\title{
CentrifugationSynchronization Control of Fractional Order Chaotic Systems Based on Memristor and Its Hardware Implementation
}

\author{
Zhaohan zhang, Huiling Jin \\ Zhoukou Vocational and Technical College, Henan, China
}

\begin{abstract}
:
This paper studies the synchronization control of fractional order chaotic systems based on memristor and its hardware implementation. This paper takes the complex dynamic phenomena of memristor turbidity system as the research background. Starting with the integer order memristor system, the fractional order form is derived based on the integer order turbid system, and its dynamics is deeply studied. At the same time, the turbidity phenomenon is applied to the watermark encryption algorithm, which effectively improves the confidentiality of the algorithm. Finally, in order to suppress the occurrence of turbidity, a fractional order sliding mode controller is proposed. In this paper, the sliding mode controller under the function switching control method is established, and the conditions for the parameters of the sliding mode controller are derived. Finally, the experimental results analyze the stability of the controlled system under different parameters, and give the corresponding time-domain waveform to verify the correctness of the theoretical analysis.
\end{abstract}

Keywords: Memristor, Fractional Order Chaotic System, Synchronization Control, Sliding Mode Controller.

\section{INTRODUCTION}

The concept of memristor was first proposed by Chinese American scientist Cai Shaotang in 1971. On the premise of the nonlinear relationship between charge and magnetic flux, he proposed the theory that memristor is the fourth basic element in circuit [1-2]. As a nonlinear element with memory function, memristor can remember the number of charges flowing through it, change its resistance by controlling the change of current, and the change characteristics of current can still be maintained in case of power failure. It is a new type of 
Article History: Received: 28 October 2021 Revised: 05 December 2021 Accepted: 10 January 2022 Publication: 28 February 2022

passive electronic device that neither generates power gain nor stores energy and consumes energy [3]. Its discovery opens up a new channel for electronic circuits and is expected to explore some new functions [4].

Lorenz discovered the first chaotic system, Lorenz system, when studying the simplified model of atmospheric convection in 1963 [5-8]. Later generations studied the chaos theory on this basis. Chaos reveals the unity of order and disorder, and the unity of certainty and randomness. It refers to the similar random phenomenon in the deterministic system. The establishment of chaos theory has changed people's view of nature. It makes determinism and probability theory, two seemingly unrelated subject systems, related. In addition, there are some phenomena in memristor oscillation circuit, such as long transient chaotic process under global stable periodic oscillation and hyperchaos with complex transient transfer behavior. Therefore, memristor chaotic system has become an important research hotspot in the field of nonlinear circuits.

\section{DYNAMIC ANALYSIS OF FRACTIONAL MEMRISTOR CHUA SYSTEM AND ITS APPLICATION}

1.Stability analysis of Chua system with fractional memristor

The system can be written in a general nonlinear form [9-10]:

$D^{q} x=f(x)(1)$

The equilibrium point of system (1) can be calculated by solving the following equation:

$$
D^{q} x=0 \Leftrightarrow f\left(x_{e q}\right)=0 \text { (2) }
$$

According to Lyapunov indirect method (Lyapunov first method), the stability of the nonlinear system can be obtained by studying the corresponding equilibrium point of the linear system, and the nonlinear system is linearized near the equilibrium point as follows:

$$
D^{q} x=J x(0<q<1)
$$

Of which:

$$
J=J\left(\frac{\partial f}{\partial x}\right) x_{e q}=0
$$

$\mathrm{J}$ is the Jacobian matrix of $\mathrm{x}$ at $\mathrm{xe}_{\mathrm{q}}=0$.

Next, for the obtained linear system (4), there are the following lemmas, which are given and proved in the literature:

For fractional order linear system (4), if all eigenvalues $\lambda$ of Jacobian matrix $J$ satisfy the condition:

$$
\min _{l \leq i \leq 4}\left|\arg \left(\lambda_{i}\right)\right|>q \frac{\pi}{2}(5)
$$


Article History: Received: 28 October 2021 Revised: 05 December 2021 Accepted: 10 January 2022 Publication: 28 February 2022

Then the system is asymptotically stable.

If the fractional order linear system (4) is asymptotically stable, its corresponding fractional order nonlinear system is also asymptotically stable at the equilibrium point $\mathrm{x}_{\mathrm{eq}}=0$.

After calculation, the Jacobian matrix $\mathrm{J}$ of the fractional memristor chaotic system proposed in this chapter at $\mathrm{Xeq}=0$ has a zero eigenvalue, a positive real part eigenvalue and a complex conjugate eigenvalue of two negative real parts:

$$
\left\{\begin{array}{l}
\lambda_{1}=0 \\
\lambda_{2}=3.2867 \\
\lambda_{3}=-0.7830+2.3434 i \\
\lambda_{4}=-0.7830-2.3434 i
\end{array}\right.
$$

2. Application of fractional memristor chaotic system in watermark encryption

At present, chaotic system and wavelet transform are more and more widely used in watermark technology, so a watermark encryption algorithm based on fractional memristic chaotic system and discrete wavelet transform is proposed. The principle is to encrypt the watermark by using the chaotic sequence generated by the fractional Chua system, and then embed the processed watermark information into the image to be encrypted by discrete wavelet transform.

In the process of image processing, the compression process of image will lead to the damage of digital watermark. Therefore, the embedding and extraction of watermark must have high robustness against compression damage in the process of watermark encryption. Discrete wavelet transform (DTW) is a local transform, which has the ability of multi-scale analysis. Through wavelet transform, the original image sequence can be decomposed into multi frequency sub images that can adapt to human visual characteristics.

A two-dimensional image can be decomposed into components of different frequencies, and the image can be decomposed into four parts in each level of decomposition. For example, the first level decomposition: $\mathrm{LL}_{1}, \mathrm{LH}_{1}, \mathrm{HL}_{1}, \mathrm{HH}_{1}$, where $\mathrm{LL}_{1}$ is the low-frequency component part, which contains the contour edge of the image, and $\mathrm{LH}_{1}, \mathrm{HL}_{1}, \mathrm{HH}_{1}$ is the high-frequency part, which contains the detail part of the image. The low-frequency part $\mathrm{LL}_{1}$ can continue to be decomposed into $\mathrm{LL}_{2}, \mathrm{LH}_{2}, \mathrm{HL}_{2}$ and $\mathrm{HH}_{2}$. Repeat this process until the required decomposition level $\mathrm{LL}_{\mathrm{n}}$ is obtained.

Let the size of the grayscale image I to be encrypted be $\mathrm{N} \times \mathrm{N}$. The embedding steps are:

Step 1: the image $\mathrm{I}$ is decomposed into four overlapping bands $\mathrm{LL}_{2}, \mathrm{LH}_{2}, \mathrm{HL}_{2}$ and $\mathrm{HH}_{2}$ by two-level discrete wavelet decomposition, and the size of the embedded watermark $\mathrm{W}$ is determined to turn $\mathrm{W}$ into a binary image;

Step 2: the chaotic sequence generated by the fractional memristor system is used to form a chaotic image M. the encrypted watermark image obtained by XOR processing the binary 
Article History: Received: 28 October 2021 Revised: 05 December 2021 Accepted: 10 January 2022 Publication: 28 February 2022

chaotic image $\mathrm{m}$ and the binary watermark image $\mathrm{W}$ is called $\mathrm{U}$;

Step 3: embed the encrypted watermark image $\mathrm{u}$ into the original image $\mathrm{I}$ according to formula (7):

$$
I_{2}^{\prime}(i, j)=I_{2}^{\square}(i, j)+\alpha U(i, j)
$$

Among $\alpha \in[0,1]$ Is the visual factor, and $I_{2}(i, j)$ represents the second-order wavelet decomposition coefficient.

Step 4: perform the inverse transformation (IDWT) of two-level DWT on $\mathrm{I}_{2}(\mathrm{i}, \mathrm{j}$ ) to obtain the wavelet coefficient $I_{2}(i, j)$ of each part, and then combine the four parts into an encrypted image y containing the original image information.

The watermark extraction process is the inverse process of embedding. The specific steps are as follows:

Step 1: perform two-level DWT decomposition on the encrypted image y and extract the wavelet coefficients $I_{2}(i, j)$ of all parts;

Step 2: perform secondary DWT decomposition on the original image I;

Step 3: calculate the wavelet coefficient of the encrypted watermark image $\mathrm{u}$ according to formula (8):

$$
U^{\prime}(i, j)=\left(\stackrel{\dot{I}}{I_{2}^{\prime \prime}}(i, j)-\stackrel{\bullet}{I}(i, j)\right) / \alpha(8)
$$

Step 4: the watermark image $\mathrm{W}$ is extracted by using the chaotic sequence generated by the fractional memristor chaotic system in the embedding algorithm. At this time, if the generated chaotic sequence is different from that in the embedding algorithm, the watermark image cannot be extracted, so the key can be set here.

The overall design flow chart of the proposed algorithm is shown in Figure 1: 
Article History: Received: 28 October 2021 Revised: 05 December 2021 Accepted: 10 January 2022 Publication: 28

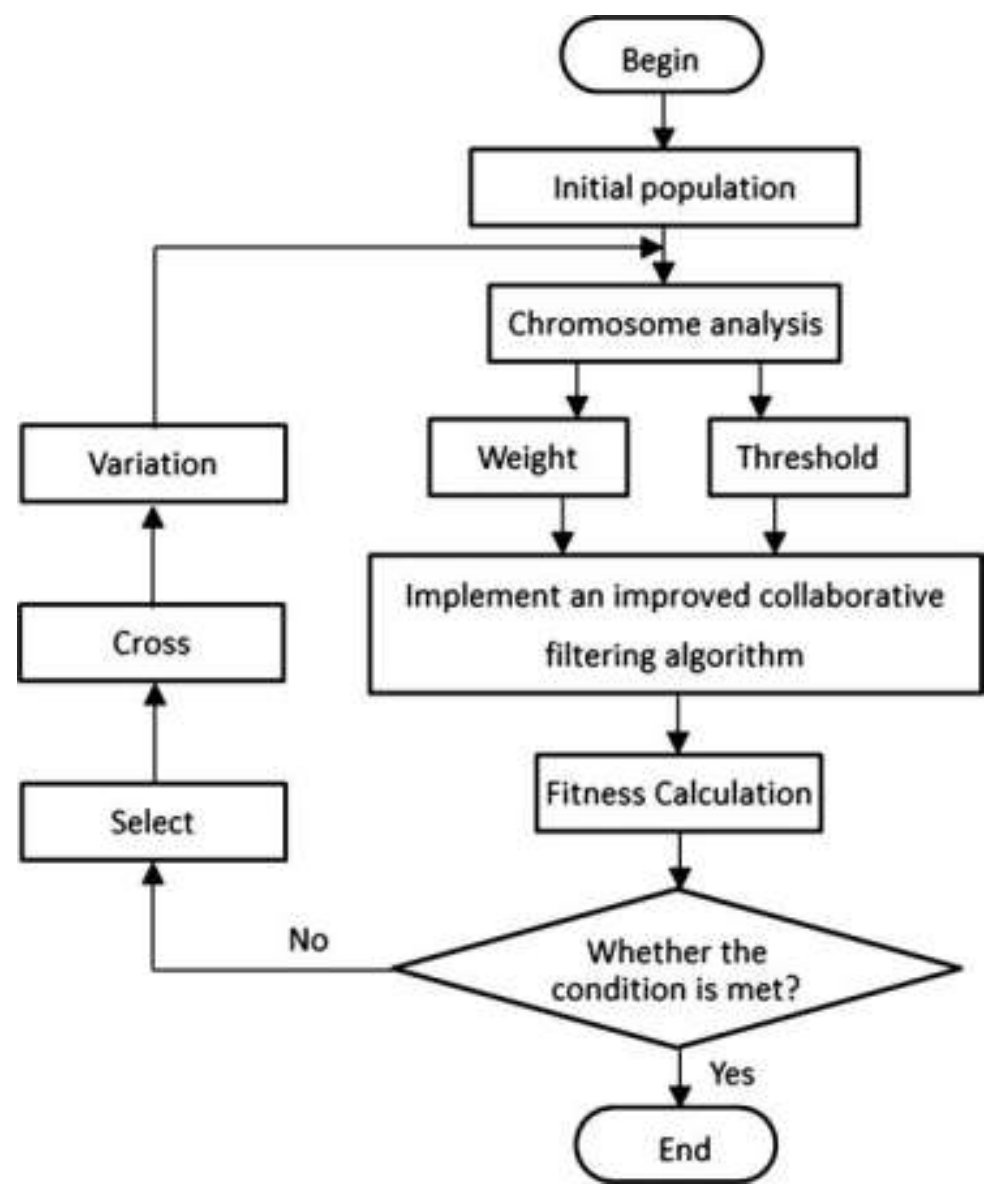

Fig 1: Algorithm flow chart

\section{SLIDING MODE CONTROL OF FRACTIONAL ORDER MEMRISTOR CHUA SYSTEM}

1.Design of sliding mode controller

Sliding mode control is a variable structure control method that uses high-frequency conversion control to change the dynamic characteristics of nonlinear systems. It is a control with sliding mode motion, and its state feedback control law is not a function of time continuity. The position in the current state space will switch from one continuous structure to another, and the trajectory will always move in the direction of the switching condition. The steps of sliding mode control are:

(1) Select the appropriate sliding surface s (t). The sliding mode surface, also known as the switching function, can make the system have the desired dynamic behavior;

(2) Select the appropriate control law U. The control law can make every point on the 
Article History: Received: 28 October 2021 Revised: 05 December 2021 Accepted: 10 January 2022 Publication: 28 February 2022

sliding surface have sliding modes.

At present, the selection of sliding mode surface mainly includes linear sliding mode surface, integral sliding mode surface, piecewise sliding mode surface, fuzzy sliding mode surface and moving sliding mode surface. The most commonly used are linear sliding surface and integral sliding surface.

(1) Linear sliding surface. The structure of linear sliding mode surface is called the simplest and most practical structure for establishing sliding mode control system. The standard linear sliding mode surface is generally designed as follows:

$$
s(t)=C x(t)(9)
$$

According to the linear system theory, the traditional design method changes the selection of sliding mode surface into the selection of coefficient matrix $\mathrm{C}$. The coefficient matrix $\mathrm{C}$ can be determined by pole assignment and the optimal quadratic performance index to meet the dynamic characteristics such as the stability of sliding mode.

(2) Integral sliding surface. When the system has uncertain parameters or some disturbances, the sliding mode of the system should remain unchanged, which is an important feature of sliding mode control. However, considering that the system does not have this property in the approach stage, how to eliminate the approach motion and make the system robust has become the focus of the integral sliding mode research.

The integral sliding surface is generally designed as follows:

$$
s(t)=C D^{q-1} x(t)(10)
$$

According to the corresponding stability theory, the value of control coefficient matrix $\mathrm{C}$ can be determined, and the specific form of sliding mode surface to be designed can be obtained

The premise of sliding mode control is that the controlled system can reach the sliding mode surface, that is, the designed controller should meet the reachability. In the design process, generally speaking, the faster the approach speed is, the better. However, if the approach speed is blindly increased, the system chattering will be severe and the control effect will be reduced accordingly. Generally, there are two forms of sliding mode controller design:

(1) Constant value switching control:

$$
U=u_{0} \operatorname{sign}(s)(11)
$$

$\mathrm{u}_{0}$ is the control parameter.

(2) Function switching control:

$$
U=u_{e q}+u_{0} \operatorname{sign}(s)(12)
$$

Where $\mathrm{u}_{\mathrm{eq}}$ is equilibrium control, also known as equivalent control, which keeps the state trajectory in the switching surface.

The commonly used switching control laws are designed in the following ways:

(1) Constant velocity control law: 
Article History: Received: 28 October 2021 Revised: 05 December 2021 Accepted: 10 January 2022 Publication: 28

February 2022

$$
\stackrel{\ominus}{s}=\varepsilon \cdot \operatorname{sign}(s)(13)
$$

Where sign (s) is a symbolic function, $\varepsilon$ is constant and $\varepsilon>0$. Selecting the appropriate control coefficient can make the system achieve good control effect.

(2) Exponential control law:

The exponential control law is mainly designed to make the state point far from the switching surface reach the switching surface at an exponential change speed:

$$
\stackrel{\forall}{=}=-\operatorname{sign}(s)-k s
$$

(3) Power control law

Power control law is to make the state point reach the switching surface in power form. It can ensure that the state point reaches the switching surface within a certain time. Its advantage is that it can eliminate the chattering caused by inertia:

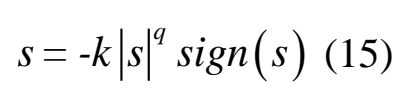

Where $\mathrm{k}>0,0<\mathrm{q}<0$.

(4) Fractional order sliding mode control law

$$
D^{q} \stackrel{\forall}{=}=-k \operatorname{sign}(s)(16)
$$

Where $\mathrm{k}>0,0<\mathrm{q}<0$, the control is completed by adjusting the control parameters, changing the approaching speed and the value of sliding mode surface derivative $s$.

2.Stability analysis of controlled system

According to the discussion in the previous section, the designed controller has met the existence and arrival conditions. Next, the stability conditions are discussed.

If the appropriate gain matrices $\mathrm{B}, \mathrm{C}$ and control gain $\mathrm{m}$ are selected so that $\mathrm{m}<0$ and $\mathrm{C} \cdot \mathrm{B}>0$, the controlled system is asymptotically stable.

It is proved that the Lyapunov function is:

$$
V_{S M C}=\frac{1}{2} s^{2}
$$

By deriving equation (17), we obtain:

$$
\begin{aligned}
& V_{S M C}=s \stackrel{\text { 보 }}{ } \\
& =s C D^{q} X(t) \\
& =s C\left[A X+H(X)+B\left(u_{e q}+\operatorname{msign}(s)\right)-H(X)\right] \\
& =s\left[C A X(t)-C \cdot B(C \cdot B)^{-1} C A X(t)+C \cdot B \operatorname{mign}(s)\right] \\
& =\operatorname{sign}(s) C \cdot B
\end{aligned}
$$

According to the Lyapunov stability theorem, in the above formula, when $\mathrm{m}<0$ and $\mathrm{C} \cdot \mathrm{B}>$ 0 , because ssign $(\mathrm{s})>0$, there is $\mathrm{V}_{\mathrm{SMC}}<0$ for $\mathrm{S}(\mathrm{T}) \neq 0$, that is, the system can reach the sliding mode surface in a finite time. When the system reaches the sliding surface: 


$$
\begin{aligned}
& D^{q} X(t)=A X(t)+H(X(t))+B\left(u_{e q}+\operatorname{msign}(s)\right)-H(X) \\
& =\left(E-B(C \cdot B)^{-1} C\right) A X+B \operatorname{msign}(s)
\end{aligned}
$$

set up:

$$
A_{S M C}=\left(E-B(C \cdot B)^{-1} C\right) A(20)
$$

\section{CONCLUSION}

The emergence of memristor provides a new direction for chaos research. It is proved that the chaotic system based on memristor is not only conducive to the dynamic analysis of nonlinear system, but also produces more complex chaotic signals. With the concept of fractional order becoming well known, the application of memristor system in fractional chaotic system has become a more innovative research direction. Based on the fractional Chua system, a new fractional memristor system is proposed by replacing the diodes in the traditional Chua system with memristors. Through the dynamic analysis and numerical simulation of the system, it can be proved that the new system has chaotic behavior. At present, memristor system has been a hot research topic in academic circles. The theoretical research is becoming more and more mature, but it is not mature in practical application. Some practical applications should become the research trend of memristor chaotic system. For the control field of memristor chaotic systems, the key control theory and technology is still a challenging research topic.

\section{ACKNOWLEDGEMENTS}

This research is supported by the key scientific research projects of colleges and universities in Henan Province (Grant No. 20B413011.). This study was supported by the science and technology public relations project of Henan Province (Grant No. $192102210250)$

\section{REFERENCES}

[1] Liu, B. J. (2005). Consideration on improving the efficiency in open-stack borrowing. Journal of Baoding Teachers College, 35(6), 350-5.

[2] Aoyama, H., Nakai, T., \& Ito, S. (1987). 5063 selecting stay place and seats of library users' : a study on planning of open stack area in public libraries based on users' activities part 3. UNEP Regional Seas Reports and Studies (UNEP). no. 84., 122(1), 155-166.

[3] Bennett, M. J., Buxton, D. T., \& Capriotti, E. (1979). Shelf-reading in a large, open-stack library. Journal of Academic Librarianship, 5(1), 4-8. 
Article History: Received: 28 October 2021 Revised: 05 December 2021 Accepted: 10 January 2022 Publication: 28 February 2022

[4] L. De Giovanni, G. Massi, \& F. Pezzella. (2013). An adaptive genetic algorithm for large-size open stack problems. International Journal of Production Research, volume 51(3), 682-697.

[5] Li-Yao, L. I., You, Y., Zhao, S. K., \& Hua-Rong, X. U. (2015). Dynamic bandwidth allocation strategy based on open stack cloud platform. Journal of Minnan Normal University.

[6] Rui, K. (2015). Research on cloud computing system based on open stack platform. Journal of Chongqing University of Science \& Technology.

[7] Xi, S., Li, C., Lu, C., \& Gill, C. D. (2015). Rt-open stack: cpu resource management for real-time cloud computing. 179-186.

[8] Jalali, Mansour, et al. "The Effect of Cloud Computing Technology in Personalization and Education Improvements and its Challenges." Procedia - Social and Behavioral Sciences 83.3(2013):655-658.

[9] Khayyambashi, M. R., \& Shahrokhi, E. (2016). Design and implement a new cloud security method based on multi clouds on open stack platform. , 6(2).

[10] Nirmal, D. A., Arunkumar, K., Girish, V., \& Arunkumar, R. (2014). Setting up based private cloud using open stack. Networking \& Communication Engineering. 